\title{
Molecular cloud fragmentation and core collapse
}

\author{
Shantanu Basu \\ Department of Physics and Astronomy, The University of Western Ontario, \\ London, Ontario N6A 3K7, Canada \\ email: basu@uwo.ca
}

\begin{abstract}
I review some steps in the conversion of molecular cloud gas into stars and planets, with an emphasis in this presentation on the early stage molecular cloud fragmentation that leads to elongated filaments/ribbons. Magnetic fields can play a crucial role in all stages and need to be invoked particularly for early stage fragmentation as well as in late core collapse where it may control disk formation. I also review some elements of hydrodynamic modeling of disk evolution.
\end{abstract}

Keywords. ISM: clouds, ISM: magnetic fields, ISM: molecules, MHD, stars: formation

\section{Introduction}

The conversion of molecular gas into stars and planets can for simplicity be conceptually divided into four stages: (1) the fragmentation of clouds into large scale structures (filaments/ribbons); (2) the formation of dense cores within these large structures; (3) core collapse to form a hydrostatic protostar; (4) disk formation and evolution leading to outflows, multiplicity, planets, etc. In this review I emphasize step (1), given recent developments in the literature, and comment briefly on the others.

\section{Cloud fragmentation and filaments}

Molecular clouds are assembled from H I clouds in the interstellar medium that have a mass-to-flux ratio $M / \Phi$ that is significantly less than the critical value required for gravitational fragmentation and collapse (Heiles \& Troland 2005). If molecular clouds are assembled from these subcritical components, then the observed star formation in the clouds leads to two possibilities: (1) the molecular clouds are entirely supercritical, having been assembled from a sufficient amount of subcritical material, by motions along Galactic magnetic field lines; (2) the molecular clouds are largely subcritical but that the breakdown of flux freezing due to ambipolar diffusion allows subregions to become subcritical and produce dense cores. The first scenario places challenging demands on the accumulation length of matter in molecular clouds, and also on the speed of streaming motions along the magnetic field (Mestel 1999). The second scenario has the drawback that the ambipolar diffusion timescale is at least $10^{7} \mathrm{yr}$, or even much longer, in diffuse molecular gas. However, the addition of turbulent initial conditions leads to the formation of dense structures on a much shorter turbulent crossing time. Within the compressed gas, rapid ambipolar diffusion can take place due to the elevated density and magnetic field gradient. This scenario has been explored in many studies over the last decade (Nakamura \& Li 2005; Elmegreen 2007; Kudoh \& Basu 2008; Nakamura \& Li 2008; Basu et al. 2009; Kudoh \& Basu 2011) and also provides a natural way to understand filamentary structures in clouds. By filaments we do not mean objects with cylindrical symmetry, 


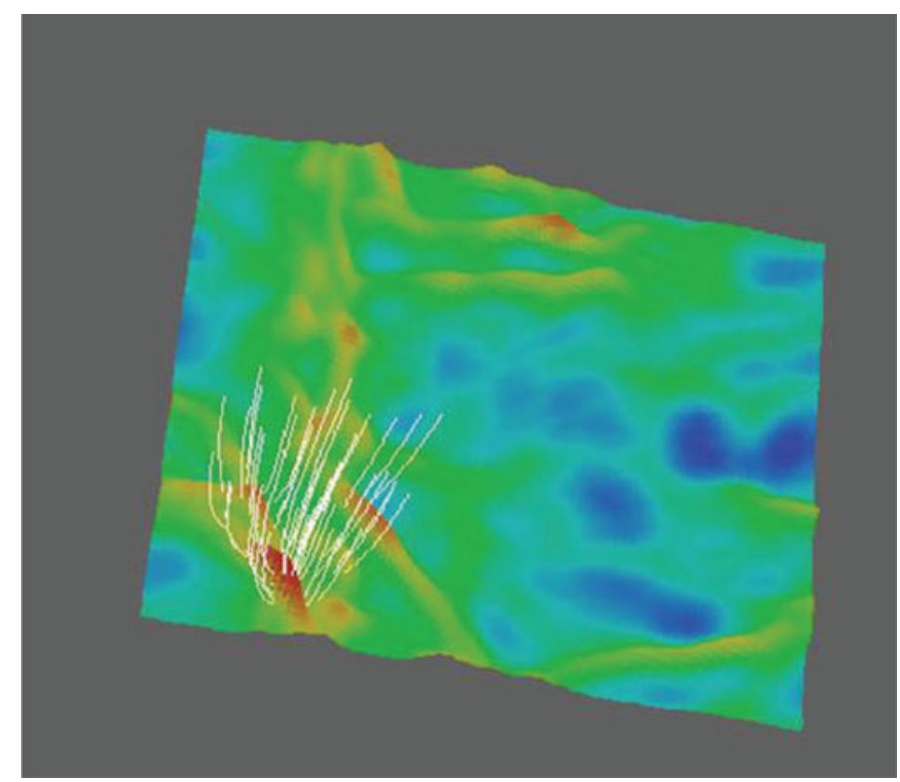

Figure 1. An image of column density in a thin sheet perturbed with turbulent initial conditions. There is a large scale magnetic field initially perpendicular to the sheet, and is illustrated with white lines in the maximally compressed region only. Turbulence and magnetic fields lead to elongated structures (ribbons) with magnetic field largely perpendicular to the long axis of the ribbon. From a simulation by Basu et al. (2009).

but rather more ribbon-like structures, with flattening along the ambient magnetic field direction, and compression by turbulence in primarily one direction perpendicular to the magnetic field. The overall scenario is illustrated in Figure 1, taken from a simulation of Basu et al. (2009). The filament formation is thus quite distinct from the cosmological filament formation simulations (e.g., Springel et al. 2005). in which the gravitational field of cold dark matter is able to collapse into filamentary structures on a local dynamical time. The magnetic turbulent picture allows the formation of filaments but not necessarily in a volume filling network as in cosmological simulations, since large parts of clouds may remain in a subcritical common envelope with little to no star formation. Models of magnetohydrodynamic (MHD) wave propagation in inhomogeneous clouds (Kudoh \& Basu 2003; Kudoh \& Basu 2006) also show that the velocity dispersion is essentially Alfvénic and that low density regions with high local Alfvén speed support large amplitude velocity fluctuations, in agreement with the observed linewidth-size relations of molecular clouds (Solomon et al. 1987). Magnetic fields can also lower the dissipation rate of turbulence in some cases, although not necessarily dramatically (Kim \& Basu 2013), and allow more efficient distribution of outflow power across the cloud (Wang et al. 2010).

Recent observations by the Herschel Space Observatory have established that the dense cores and young stellar objects are often threaded along networks of elongated structures seen in projection (André et al. 2010; Men'schikov et al. 2010; Arzoumanian et al. 2011). When magnetic field morphology can be detected through polarized dust emission, the magnetic field is generally aligned perpendicular to the long axis of the filaments (Palmeirim et al. 2013). These features are at least qualitatively consistent with the magnetic turbulent scenario described above. Another interesting result is that the measurement of average FWHM widths across filaments in three different clouds shows 
a cluster of values around $\sim 0.1 \mathrm{pc}$, even though the Jeans length calculated from the peak column densities are varying by about two orders of magnitude (Arzoumanian et al. 2011).

It seems that the filament widths require an explanation that goes beyond the simple Jeans length prescription. They are likely to be quasi-equilibrium objects based on the amount of fragmentation along their lengths to form dense cores. Recall that for a nonmagnetized isothermal cylinder, there is a critical line mass $2 c_{s}^{2} / G$ (where $c_{s}$ is the isothermal sound speed) below which radial equilibrium is possible but fragmentation occurs along the axis of the cylinder. However if the line mass exceeds this critical value, then indefinite collapse to a spindle occurs before any fragmentation can take place (Inutsuka \& Miyama 1997). Modified values of critical line mass, actually critical line mass to line flux ratio, for magnetized cylinders with a lateral magnetic field have recently been obtained by Tomisaka (2014) and Hanawa \& Tomisaka (2015). Given the fragmentation along their length, it is likely that the line mass to line flux ratio of observed ribbons is subcritical.

Hennebelle \& André (2013) developed an analytic model of filament width in which the energy input from accretion onto a filament is balanced by energy dissipation by ion-neutral friction, and there is mechanical equilibrium between gravity and internal turbulence. They obtained a mean filament width of $\sim 0.1 \mathrm{pc}$ for a range of column densities. An alternate model of filament formation was explored by Kudoh \& Basu (2014) in which a filament (really ribbon) is created by turbulent compression of subcritical gas and halts contraction when magnetic pressure balances ram pressure. However, their semi-analytic model was used to estimate time scales for core formation and not to estimate filament widths.

\section{Cores to star-disk systems}

Once a dense core is formed by fragmentation of a larger clump or filament, it will begin runaway collapse as long as its mass-to-flux ratio is supercritical. The hydrodynamic collapse leads to a well known set of outcomes: development of an $r^{-2}$ density profile and a shrinking central flat density peak that eventually forms the first hydrostatic core, later collapsing to the second core of stellar dimensions (e.g., Larson 1969). The addition of rotation and/or magnetic fields leads to a flattened collapsing core, especially at high densities. Once a central protostar is formed at the pivotal moment $t=0$, several interesting things happen. An expansion wave moves outward from the center (Shu 1977), changing the character of flow variables within it. When rotation is added, a centrifugal disk is able to form, however an additional flux-frozen magnetic field leads to catastrophic magnetic braking that shuts off disk formation (Allen et al. 2003; Galli et al. 2006).

A straightforward solution to catastrophic magnetic braking is the presence of nonideal MHD effects, notably ambipolar diffusion and Ohmic dissipation. These will weaken the magnetic field strength and reduce the effectiveness of magnetic braking. In Dapp \& Basu (2010) and Dapp et al. (2012) we used the thin-disk approximation to follow collapse to the formation of a second stellar core while including non-ideal MHD effects. Both studies show that non-ideal MHD effects allow the formation of a disk immediately after the pivotal moment $t=0$. In Dapp et al. (2012) we used a chemical network model to calculate the ionization fraction and coefficients of ambipolar diffusion and Ohmic dissipation. Figure 2 shows a series of column density profiles at various times, with the last one at the moment immediately following the formation of a second core of stellar dimensions. Each distinct region of the column density profile is captured, including the MHD accretion shock created by outward moving magnetic flux (Li \& McKee 1996; Contopoulos et al. 1998). Figure 3 shows the magnetic field lines in cases with ideal 


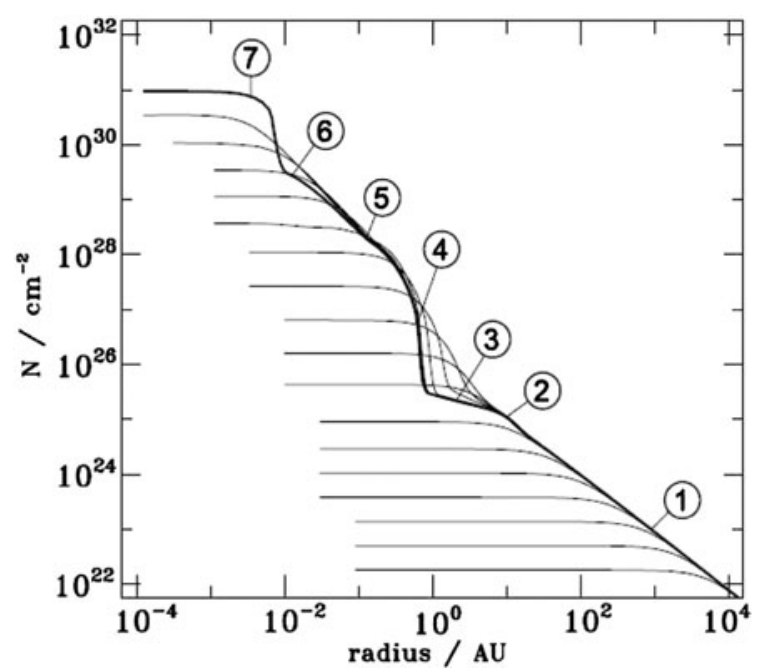

Figure 2. Column number density $(N)$ profile versus radius from Dapp et al. (2012). The thin lines are profiles in ascending order of time. Several features are identifiable via their associated breaks in the profile (1) Prestellar infall profile with $N \propto r^{-1}$. (2) Magnetic wall at $\approx 10 \mathrm{AU}$, where the bunched-up field lines decelerate material before it continues the infall. (3) Expansion wave profile with $N \propto r^{-1 / 2}$ outside the first core. (4) First core at 1 AU. (5) Infall profile onto the second core with $N \propto r^{-1}$. After the first core has reached $\approx 1,000 \mathrm{~K}$, it starts to collapse, as $\mathrm{H}_{2}$ is dissociated. (6) Expansion wave profile with $N \propto r^{-1 / 2}$ outside the second core. (7) Second core at $\approx 1 R_{\odot}$.

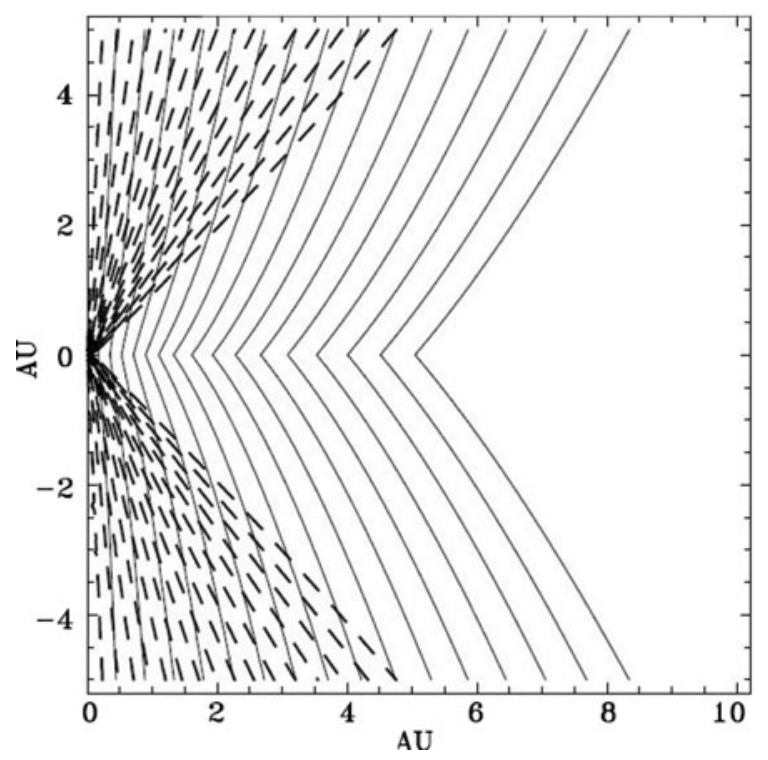

Figure 3. Magnetic field lines on 10 AU scales from Dapp et al. (2012). The dashed lines represent the flux-freezing model, while the solid lines show the same field lines for the model including non-ideal MHD effects for a grain size $a_{\mathrm{gr}}=0.038 \mu \mathrm{m}$. In both cases, the second core has just formed and is on the left axis midplane. The field lines straighten out significantly on small scales in the non-ideal MHD model compared to the flux-frozen model.

and non-ideal MHD. The former case has extreme flared field lines and no disk is able to form due to catastrophic magnetic braking. The non-ideal MHD model yields the 


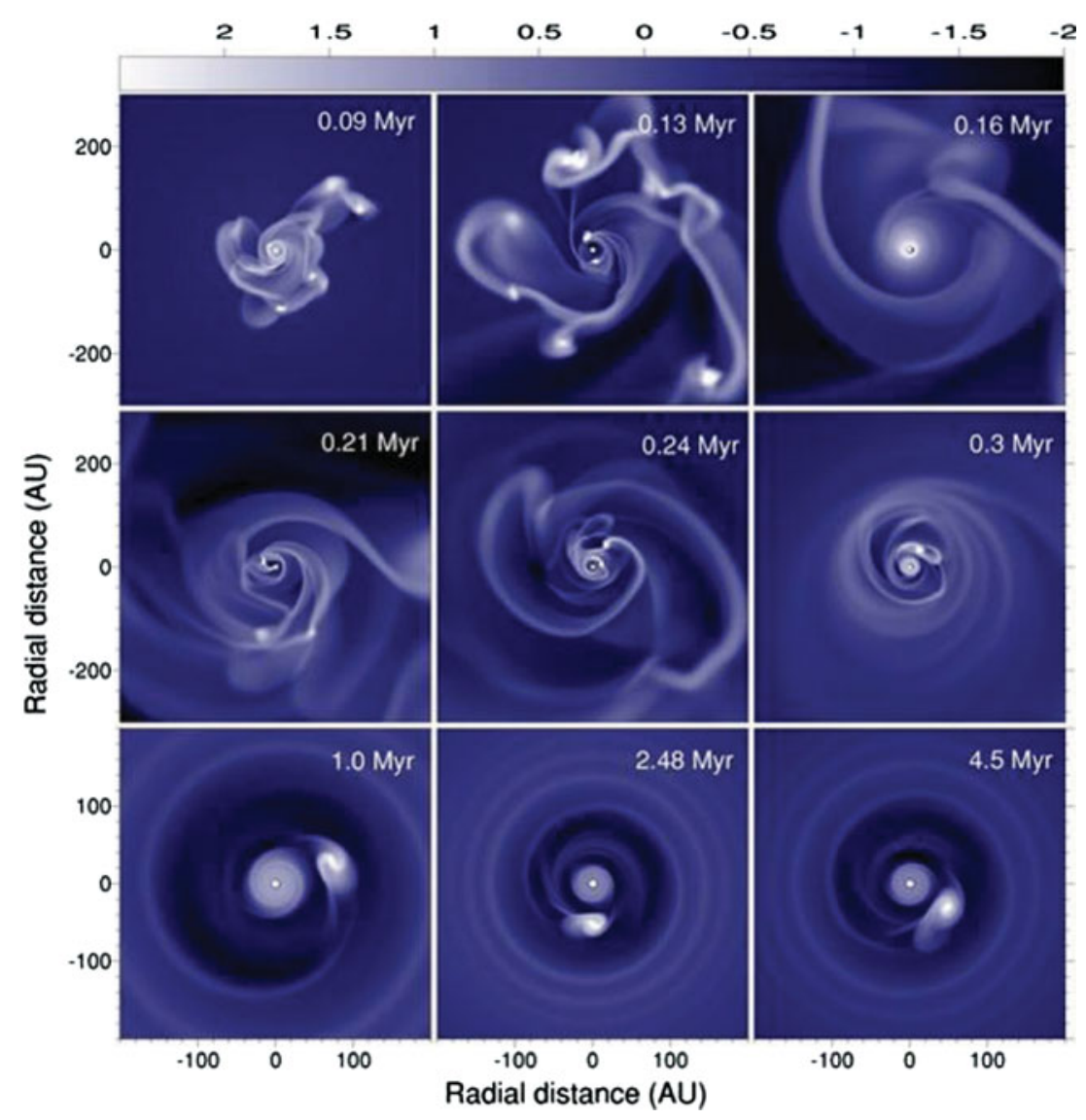

Figure 4. Gas surface density maps $\left(\mathrm{g} \mathrm{cm}^{-2}, \log\right.$ units) at six times after the formation of the central star (bright circle in the coordinate center), from Vorobyov \& Basu (2010a). Note the zoom-in as the time increases. The top two rows contain images of size $600 \mathrm{AU}$ on each side, while the bottom row contains images of size $400 \mathrm{AU}$ on each side.

development of a centrifugal disk immediately after the formation of the second core, however the loss of some angular momentum due to magnetic braking is expected to keep disks at relatively small sizes $\lesssim 10$ AU in the early Class 0 phase $\left(\lesssim 4 \times 10^{4} \mathrm{yr}\right)$.

\section{Disks and multiplicity}

As shown in the previous section, the collapse of a prestellar core leads to a very focused collapse with a power-law density profile. As a result, the probability of fragmentation during the runaway collapse phase is low. However, a centrifugally supported disk can form after a central protostar has formed. The existence of a central point mass potential allows a centrifugal barrier to be encountered by an infalling rotating fluid element that conserved its angular momentum (see discussion in Basu \& Mouschovias 1995). A protostellar disk is a quasi-equilibrium structure and evolves on a time scale much longer than its dynamical time. Therefore, it has time for fragmentation if conditions are suitable, e.g., the criteria for gravitational instability and fragmentation are satisfied (Toomre 1981). Disk fragmentation is the most likely mechanism for the formation of multiple stellar systems and the formation of brown dwarfs and giant planets at wide orbits of several tens of AU. We show here the results of hydrodynamic numerical simulations of 
Vorobyov \& Basu (2010a), who modeled the formation of the disk from the collapse of a prestellar core, as well as its subsequent evolution on Myr time scales. Figure 4 shows images of the disk column density at a series of times after formation of the central protostar. During the first $10^{5} \mathrm{yr}$, there is vigorous episodic gravitational instability in the disk, driven by mass accretion from the parent core. Clumps form in spiral arms through nonlinear gravitational instability, and are eventually driven in to the center through gravitational interaction with trailing spiral arms. In this model, one clump is able to survive to late times and eventually carves out a gap in the disk and settles into a stable orbit by $4.5 \mathrm{Myr}$. The clump is essentially a first hydrostatic core and is the progenitor of a low mass companion star with an orbit radius $\approx 52 \mathrm{AU}$. This mechanism can also produce wide orbit proto-brown-dwarfs and giant protoplanets. Details of the numerical model and the overall scenario of the migrating embryo model can be found in Vorobyov \& Basu (2006), Vorobyov \& Basu (2010b), and Vorobyov \& Basu (2015).

\section{References}

Allen, A., Li, Z.-Y., \& Shu, F. H. 2003, ApJ, 599, 363

André, P., et al. 2010 A $\mathscr{G} A, 518, \mathrm{~L} 102$

Arzoumanian, D., et al. 2011, A\&A, 529, L6

Basu, S., Ciolek, G. E., Dapp, W. B., \& Wurster, J. 2009, New Astron., 14, 483

Basu, S. \& Mouschovias, T. Ch. 1995, ApJ, 452, 386

Contopoulos, I., Ciolek, G. E., \& Königl, A. 1998, ApJ, 504, 247

Dapp, W. B. \& Basu, S. 2010, A\&A A, 521, L56

Dapp, W. B., Basu, S., \& Kunz, M. W. 2012, A\& 8 , 541, A35

Elmegreen, B. G. 2007, ApJ, 668, 1064

Galli, D., Lizano, S., Shu, F. H., \& Allen, A. 2006, ApJ, 647, 374

Hanawa, T. \& Tomisaka, K. 2015, ApJ, 801, 11

Heiles, C. \& Troland, T. H. 2005, ApJ, 624, 773

Hennebelle, P. \& André, P. 2013, A\& A, 560, A68

Inutsuka, S.-i. \& Miyama, S. M. 1997, ApJ, 480, 681

Kim, C.-G. \& Basu, S. 2013, ApJ, 778, 88

Kudoh, T. \& Basu, S. 2003, ApJ, 595, 842

Kudoh, T. \& Basu, S. 2006, ApJ, 642, 270

Kudoh, T. \& Basu, S. 2008, ApJ, 679, L97

Kudoh, T. \& Basu, S. 2011, ApJ, 728, 123

Kudoh, T. \& Basu, S. 2014, ApJ, 794, 127

Larson, R. B. 1969, MNRAS, 145, 271

Men'schikov, A., et al. 2010, A\&A, 518, L103

Mestel, L. 1999, Stellar Magnetism (Oxford: Oxford Univ. Press)

Nakamura, F. \& Li, Z.-Y. 2005, ApJ, 631, 411

Nakamura, F. \& Li, Z.-Y. 2008, ApJ, 687, 354

Palmeirim, P., et al. 2013, A\&A, 550, A38

Shu, F. H. 1977, ApJ, 214, 488

Solomon, P. M., Rivolo, A. R., Barrett, J., \& Yahil, A. 1987, ApJ, 319, 730

Springel, V. et al. 2005, Nature, 435, 629

Tomisaka, K. 2014, ApJ, 785, 24

Toomre, A. 1981, in: S. M. Fall, \& D. Lynden-Bell (eds.), The Structure and Evolution of Normal Galaxies (Cambridge Univ. Press, Cambridge), p. 111

Vorobyov, E. I. \& Basu, S. 2006, ApJ, 650, 956

Vorobyov, E. I. \& Basu, S. 2010a, ApJ, 714, L133

Vorobyov, E. I. \& Basu, S. 2010b, ApJ, 719, 1896

Vorobyov, E. I. \& Basu, S. 2015, ApJ, 805, 115

Wang, P., Li, Z.-Y., Abel, T., \& Nakamura, F. 2010, ApJ, 709, 27 